\title{
Immunofluorescent demonstration of intracellular fibrin in synovial tissue
}

\author{
R. BACH ANDERSEN AND P. ELLING
}

From the Department for Infectious Diseases, Blegdam Hospital, and the Department of Physical Medicine, Rigshospital, Copenhagen, Denmark

The presence of fibrin in and on synovial membranes has been demonstrated in previous studies by an immunofluorescence technique. This took the form of a fibrin film or an accumulation at interstitial sites (Gitlin, Craig, and Janeway, 1957; Fish, Michael, Gewurz, and Good, 1966). Fibrin inclusions have also been found in polymorphonuclear leucocytes from synovial fluids (Riddle, Bluhm, and Barnhardt, 1965). Recently, Ghadially and Roy (1967), using electron microscopy, demonstrated a fibrinlike material located intracellularly in phagocytic lining cells and extracellularly as homogeneous deposits.

The aim of the present work was to examine the extent and localization of fibrin deposits by the fluorescent antibody method, and to investigate factors which might influence the fibrin deposits (e.g. fibrin stabilizing factor (FSF) and plasminogen) in the synovial membrane of patients with rheumatoid arthritis (RA) and other types of arthritis. Furthermore, a study of the content of immunoglobulin IgG and $\beta-1 \mathrm{c}$ globulin (a component of complement) was also included.

\section{Material}

\section{PATIENTS}

Those included in the series were selected from among two groups of in-patients.

\section{Inflammatory disease (21 cases)}

This group consisted of fifteen patients with classical RA according to the criteria of the American Rheumatism Association. There were four men and eleven women aged from 22 to 66 years (average 45). Eleven patients had positive tests for rheumatoid factor. The duration of disease ranged from 2 to 18 years (average 6 ). There was one 12year-old girl with juvenile RA (ESR $26 \mathrm{~mm}$./1st hr) who had had symptoms for 7 years. There were also five patients with monoarticular arthritis (MO), three men and two women aged from 20 to 45 years (average 30). The ESR ranged from 15 to $40 \mathrm{~mm}$./1st hr (mean $25 \mathrm{~mm}$.), and the duration of symptoms from 6 to 15 months (average 10).
The patients with RA were classified into three subgroups according to the degree of disease activity: I high, II medium, and III none (Bach Andersen and Gormsen, 1970b).

\section{Noninflammatory disease (11 cases)}

There were two patients with osteoarthrosis (OA) (one 20-year-old male and one 66-year-old female, ESR 2 and $18 \mathrm{~mm}$./1st hr respectively) and nine patients with traumatic arthritis and suspected ruptured meniscus (RM), seven males and two females aged from 20 to 49 years (average 36). Their symptoms had persisted for 1 to 60 months (average 12) and the ESR ranged from 2 to 14 $\mathrm{mm} . / 1 \mathrm{st}$ hr. Synovial membranes were obtained by arthrotomy, either by synovectomy or during meniscectomy. The biopsy specimens were frozen immediately and kept at $-20^{\circ} \mathrm{C}$. until the time of examination.

\section{ANTISERA}

These were procured from the following sources:

Anti-human fibrinogen and anti-human plasminogen from Brostex, Copenhagen.

Antifibrin stabilizing factor from Behring Werke, Marburg.

Anti-human immunoglobulin and anti-human albumin from the Statens Seruminstitut, Copenhagen, and from Nordic, Tilburg, Holland.

Anti- $\beta_{1}$ globulin from Nordic, Tilburg, Holland.

All antisera were tested against the antigens (fibrinogen, plasminogen, FSF, and albumin) or normal human serum by microimmunodiffusion or microimmunoelectrophoresis according to standard micromethods. Monospecific reactions were found. In immunoelectrophoresis the anti-human immunoglobulin showed a single precipitation line (IgG) against normal human serum and was not absorbed with light chains. The antifibrinogen, antiplasminogen, and antifibrin stabilizing factor showed single precipitation lines when tested by immunodiffusion against lyophilized human plasma concentrated six times. No cross-reaction between antifibrinogen, antifibrin stabilizing factor, and antiplasminogen was demonstrated. The antisera were assayed in microimmunodiffusion against fibrinogen purified by chromatography (Sephadex 200) 
(Gormsen, 1970). This purified antigen without enzymatic contamination showed a single precipitate line with antifibrinogen and no reaction with antifibrin stabilizing factor or antiplasminogen.

\section{PLASMIN}

Porcine, lysofibrin (R), lyophilized, AS Novo, Copenhagen. Stock solution $60 \mathrm{CU}$ caseinolytic units (Sgouris) per $\mathrm{ml}$.

\section{Methods}

The direct fluorescent antibody technique, using 4-6 $\mu$ cryostat sections of synovial tissue, was employed, together with FITC-labelled antisera against fibrinogen, $\beta_{-1 c}$ globulin and human $\gamma$ globulin and albumin. The indirect immunofluorescent antibody technique was used for the immunohistochemical demonstration of plasminogen and FSF. Rabbit antisera against plasminogen and FSF were applied to the tissue, followed by application of a FITClabelled antirabbit $\gamma$ globulin. The indirect technique was also combined with the direct technique in order to obtain determination of fibrinogen. The antifibrinogen and anticomplement sera were conjugated with FITC after isolation of the IgG fraction of antisera with polyethylene glycol, followed by passage through a DEAE Sephadex column (Bergquist and Schilling, 1970) and concentration by vacuum dialysis. The IgG fraction was labelled with 16 mg. FITC per gram protein. The antihuman $\gamma$ globulin and antirabbit $\gamma$ globulin were labelled similarly after precipitation of the immunoglobulin fraction with ammonium sulphate. The synovial sections were incubated for $30 \mathrm{~min}$ at room temperature with antisera, washed twice with PBS (pH 7.2), and mounted in buffered glycerol. The slides were examined within a few hours, using a Leitz
Zernicke microscope fitted with a Tiyoda oil darkfield carcoid condenser. An iodine quartz lamp (12 volt) was used, together with a primary interference filter (400-495 $\mathrm{nm} ., 85$ per cent. transmission of light) (Rygård and Olsen, 1969 ) and a secondary filter cutting off at $530 \mathrm{~nm}$. Cryostat sections of rheumatoid synovial tissue were pretreated with plasmin for one hour at room temperature $\left(20-24^{\circ} \mathrm{C}\right.$.). The sections were washed with PBS and EACA $\left(10^{-2} \mathrm{M}\right)$ for $30 \mathrm{~min}$. and then incubated with antisera.

\section{Results}

In thirteen of the sixteen rheumatoid synovial membranes investigated, there was strong $(+++$ or more) fluorescent staining with FITC-conjugated antifibrinogen, located in the cytoplasm of the superficial layers of the synovial cells. Staining was weaker in three cases. In contrast, there was never any fluorescent staining in cells at deeper sites. Fluorescence was also observed in the homogeneous material on the synovial membranes containing cellular debris. In all cases, a more diffuse extracellular staining was seen in the subepithelial area and in small vessels which were often occluded by fibrin. This was either lining the intima or was deposited in the wall (Figs 1 and 2). There was little or no fluorescence in areas with necrosis. No significant difference was observed between the various groups of activity of RA (Table) There was no loss of fluorescence in the section treated with plasmin.

No intracellular deposits of fibrin were seen in any of the synovial membranes derived from patients with RM, MO, or OS, except in one case, viz. synovial

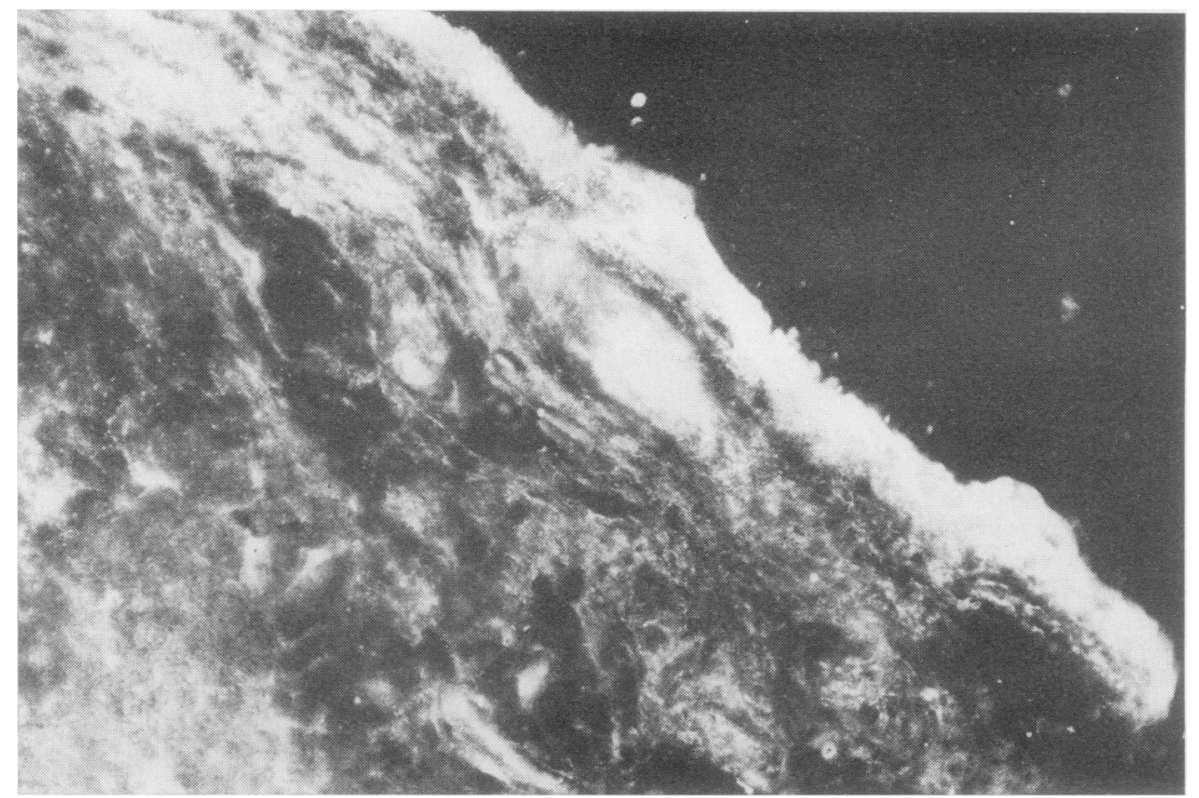

FIG. 1 Synovial membrane from a patient with $R A$ after staining with FITC-labelled antihuman fibrinogen. Accumulation of fibrinogen is seen in superficial layers of the membranes, in vessels, and as deposits at interstitial sites. $\times 100$ 


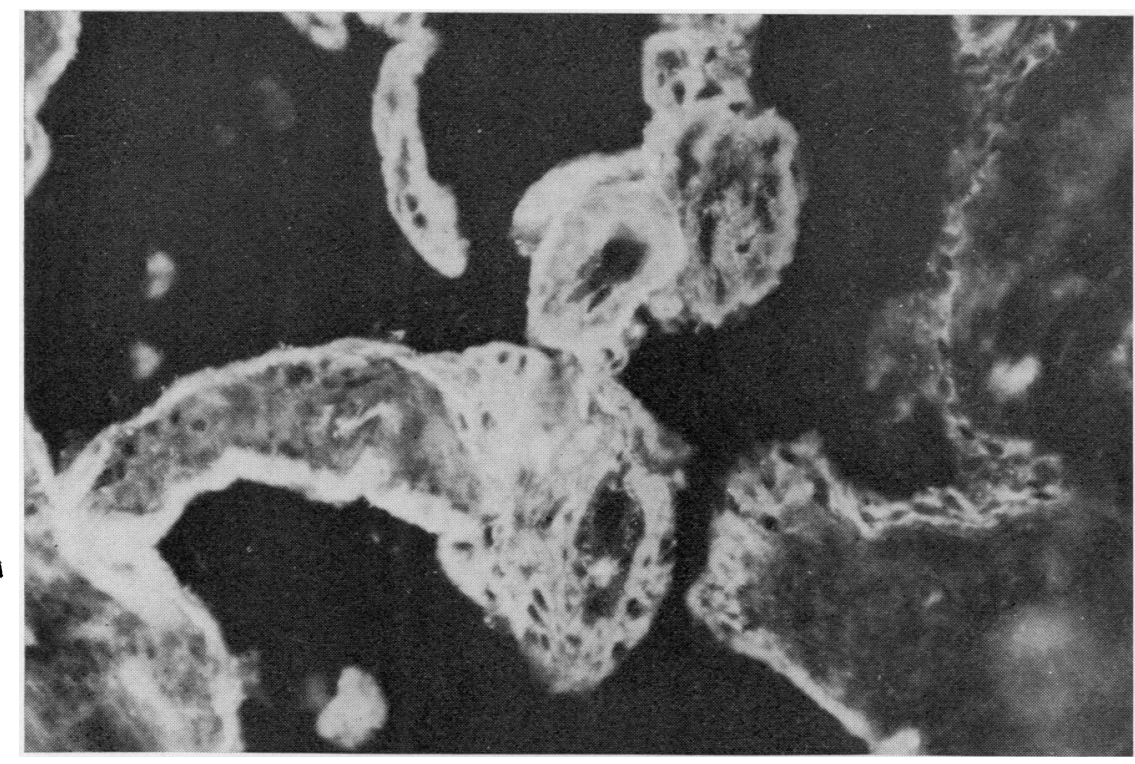

$2 a$

FIG. 2 Synovial membrane from patients with $R A$ after staining with FITC-labelled antihuman fibrinogen, showing intracellular fluorescence in synovial lining cells (a) and diffuse extracellular fluorescence (b). $\times 250$

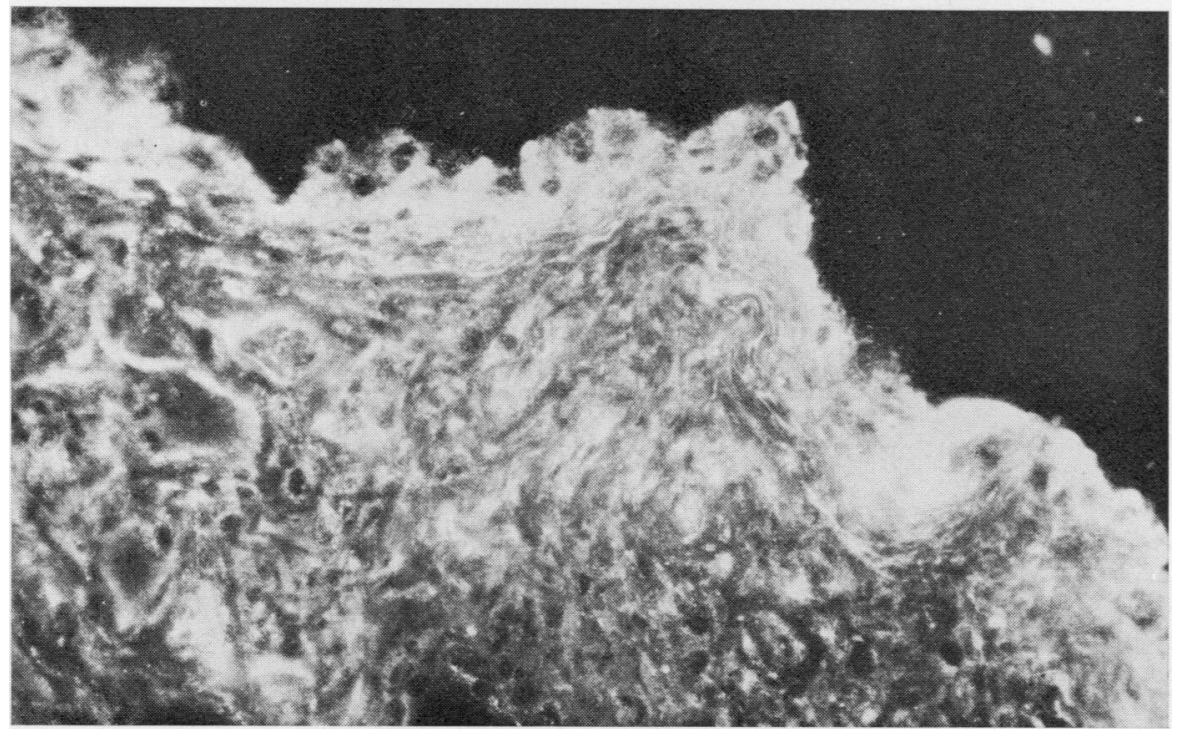

Table Survey of fluorescent staining patterns

\begin{tabular}{|c|c|c|c|c|c|c|c|}
\hline \multirow[t]{3}{*}{ Diagnosis } & \multirow{3}{*}{$\begin{array}{l}\text { No. of } \\
\text { patients } \\
\text { tested }\end{array}$} & \multicolumn{6}{|c|}{ No. of patients with positive staining } \\
\hline & & \multicolumn{2}{|l|}{ Fibrin } & \multirow[t]{2}{*}{$F S F$} & \multirow[t]{2}{*}{ Plasminogen } & \multirow[t]{2}{*}{$I g G$} & \multirow[t]{2}{*}{ Ic globulin } \\
\hline & & Intracellular & Extracellular & & & & \\
\hline 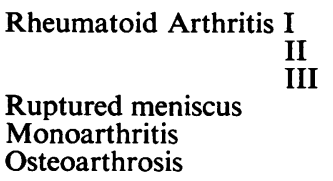 & $\begin{array}{l}4 \\
7 \\
5 \\
9 \\
5 \\
2\end{array}$ & $\begin{array}{l}4 \\
7 * \\
5 \dagger \\
0 \\
1 \\
0\end{array}$ & $\begin{array}{l}4 \\
7 * \\
5 \dagger \\
3 \\
3 \ddagger \\
0\end{array}$ & $\begin{array}{l}4 \\
7 \\
5 \\
9 \\
5 \\
2\end{array}$ & $\begin{array}{l}4 \\
7 \\
5 \\
9 \\
5 \\
2\end{array}$ & $\begin{array}{l}4 \\
7 \\
5 \\
0 \\
0 \\
0\end{array}$ & $\begin{array}{l}4 \\
7 \\
5 \\
0 \\
0 \\
0\end{array}$ \\
\hline
\end{tabular}

* Weak fluorescence only $(+,++)$ in two cases.

+ Only weak staining $(+,++)$.

$\ddagger(++)$ fibrin staining intracellularly also in one of these cases. 
membrane from a patient with MO. In six patients (three with MO and three with RM), staining was found to be weak at extracellular sites in the superficial layer (Fig. 3). No fluorescence could be seen in the vessels and in the deeper layers of the membranes in patients in this group.

In contrast to fibrin, FSF and plasminogen were found in both inflammatory and noninflammatory synovial membranes. In synovial membranes containing fibrin deposits, intracellular FSF and plasminogen could be seen on the synovial membrane, even in the homogeneous material. Furthermore, plasminogen was found in small vessels in the subepithelial area and in the vasa vasorum.

In membranes without fibrin deposits, FSF and plasminogen were seen in the superficial layers of the synovial membrane, often in groups of cells and in small vessels.

Using anti- $\beta_{-1 \mathrm{c}}$ and anti-IgG conjugates as reagents, both intracellular and extracellular fluorescence was seen in the synovial membranes in patients with RA. This was often located in single cells and there was no difference between seronegative and seropositive cases.

No fluorescence of IgG and complement was seen in the cases of noninflammatory disease and in cases of MO. None of the synovial membranes showed deposits of albumin.

After absorption of the antifibrinogen conjugate with fibrinogen, no fluorescence was observed. If the reaction of FITC-conjugated antifibrinogen was blocked by an unconjugated reagent at different intervals ( $1 \mathrm{hr}, 24 \mathrm{hrs})$, some blocking was seen after $1 \mathrm{hr}$ and almost total blocking after $24 \mathrm{hrs}$.

Synovial fluids were obtained simultaneously from six patients with RA. Sediments from these fluids gave the same immunofluorescence pattern as that observed in the homogeneous material on the synovial membranes when anti-IgG and anti- $\beta_{1 \mathrm{c}}$ conjugates were used and combined with staining with the antifibrinogen conjugate.

\section{Discussion}

In accordance with previous investigations by Gitlin and others (1957) and Fish and others (1966), the results of the present study, based on immunofluorescence, showed deposits of fibrin on the synovial membrane and at interstitial sites. The accumulation of large amounts of intracellular fibrin observed is in agreement with the electron microscopic investigations of Ghadially and Roy (1967).

The absence of albumin from the synovial membranes indicates that the fibrin deposits were not due to simple imbition of the tissue with plasma proteins. Similarly, although fibrinogen is often contaminated with plasminogen, it is not likely that such contamination would explain the findings. Plasminogen and FSF fluorescence were seen in cases of both inflammatory and noninflammatory disease, whereas fibrin staining was obtained only in synovial membranes from cases of inflammatory disease. This discrepancy in staining reactions may indicate an independen function of these factors.

The autograph fibrinolysis technique (Todd, 1964) has been used previously for the demonstration of FSF and fibrinolysis activities (tissue activator of plas minogen) (Bach Andersen and Gormsen, 1970a). By the use of a modification of this technique, FSF was found to be present, particularly in the superficial layers of the membranes and the tissue activator in the vessels of loose connective tissue in the deeper layers. This is in accordance with the localization of FSF and plasminogen demonstrated by immunofluorescence technique in the present study.

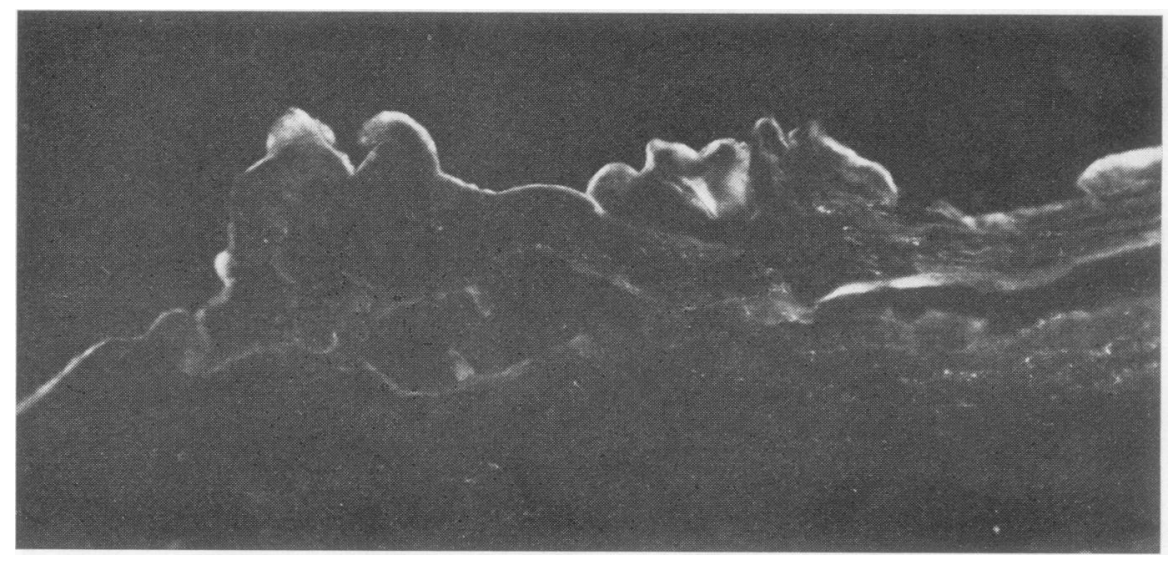

FIG. 3 Synovial membrane from a patient with noninflammatory synovial membrane after staining with FITC-labelled antihuman fibrinogen. Fluorescence is seen as a thin film on the synovial lining cells, but there is no fluorescence in layers at deeper sites. $\times 100$ 
The observation of identical immunofluorescence patterns in the homogeneous material and in sediments of synovial fluids may indicate that one and the same type of precipitate is involved. In other words, the same sediment may result from an exfoliation of the homogeneous material on the synovial membranes. This is in accordance with the findings of Albrecht, Marinetti, Jacox, and Vaughan (1965) in a biochemical and ultramicroscopical study of rice bodies from rheumatic synovial fluid.

The accumulation of large amounts of fibrin at intracellular and extracellular sites may be explained in several ways. FSF in itself did not seem to cause the fibrin to become resistant to lysis (Feddersen and Gormsen, 1971). The low activity of fibrinolysis in synovial fluid from patients with RA, which may be attributable to a high concentration of inhibitors, may result in an increased fibrin accumulation in the superficial part of the membranes (Bach Andersen and Gormsen, 1970b). However, although low fibrinolysis activity may play a certain role, the high fibrin content in the synovial lining cells may rather be caused by an altered structure of fibrin. Thus synovial fluids from patients with RA were found to have a high content of noncoagulable fibrinogen antigenic proteins-split products (Gormsen, Bach Andersen, and Feddersen, 1971). These split products may form complexes with fibrinogen and/or high molecular fibrinogen/fibrin degradation products which, in the same way as the deposits demonstrated by immunofluorescence, are more or less resistant to the action of plasmin (Gormsen and others, 1971). Hypothetically, precipitates of these complexes with immunoglobulins, polysaccharides, and other proteins may then be formed and phagocytosed by lining cells in the synovial membrane. The finding of fibrin, IgG, and complement in the same lining cells in the synovial membranes of patients with rheumatoid arthritis may thus indicate that active cellular, and possibly immunological, reactions occur in order to free the synovial environment of these precipitates.

\section{Summary}

Synovial membranes from patients with rheumatoid arthritis (16) and monoarticular arthritis (5), and from patients subjected to meniscectomy (11) have been investigated by an immunofluorescence technique for the presence of immunoglobulins, complement factors, albumin, fibrin, and factors which might influence the fibrin deposits, e.g. fibrin-stabilizing factor and plasminogen.

One of the most conspicuous results was the finding in all rheumatoid synovial membranes of large quantities of fibrin on the surface and in the cytoplasm of the synovial lining cells, whereas only small amounts of fibrin were seen lining the surface of the membranes in three patients with monoarticular arthritis and in three with traumatic arthritis (suspected ruptured meniscus). No fibrin deposits were found in the remaining ten patients. In contrast, fibrin stabilizing factor and plasminogen were demonstrated in both inflammatory and noninflammatory synovial membranes. In synovial membranes with fibrin deposits, fibrin stabilizing factor and plasminogen were seen in the same area as the fibrin, and also in the smaller vessels. In synovial membranes without fibrin deposits, fibrin stabilizing factor and plasminogen were present in the superficial layer of the synovial membranes, in the vessels, and occasionally in groups of cells in the synovial membrane.

No albumin was found in synovial cells. There were small amounts of immunoglobulins and complement factors $\left(\gamma_{1 c}\right)$ at both intracellular and extracellular sites in membranes of patients with RA, but not in noninflammatory synovial membranes.

The results show that intracellular fibrin is present in large quantities in synovial lining cells and thus is probably phagocytosed by these cells. The finding of these increased deposits of fibrin is discussed on the basis of previous results which indicated the formation of fibrin less susceptible to proteolytic enzyme (plasmin), and on results showing that the fibrinolytic activity is low in synovial fluids from patients with rheumatoid arthritis.

\section{References}

Albrecht, M., Marinetti, G. V., Jacox, R. F., and Vaughan, J. H. (1965) Arthr. and Rheum., 8, 1053 (A biochemical and electron microscopy study of rice bodies from rheumatoid patients)

Bach ANDersen, R., AND Gormsen, J. (1970a) Ann. rheum. Dis., 29, 287 (Fibrinolytic and fibrin stabilizing activity of synovial membranes)

(1970b) Acta rheum. scand., 16, 319 (Fibrin dissolution in synovial fluid)

BERgQuist, N. R., AND SCHILlING, W. (1970) 'Preparation of antihuman immunoglobulins for indirect fluorescent tracing of autoantibodies', in 'Standardization in Immunofluorescence', ed. E. J. Holborow, p. 171. Blackwell, Oxford

Bluhm, G. B., Riddle, J. M., AND Barnhart, M. J., (1966) Henry Ford Hosp. med. Bull., 14, 119 (Significance of fibrin and other particulates in rheumatoid joint inflammation)

Feddersen, C., AND GoRmsen, J. (1971) Scand. J. clin. Lab. Invest., 27, 175 (Plasmin digestion of stabilized and non-stabilized fibrin illustrated by $\mathrm{pH}$-stat titration and thrombelastography)

Fish, A. J., Michael, A. F., GewUrZ, H., AND Good, R. A. (1966) Arthr. and Rheum., 9, 267 (Immunopathologic changes in rheumatoid arthritis synovium) 
Ghadially, F. N., AND Roy, S. (1967) Ann. rheum. Dis., 26, 426 (Ultrastructure of synovial membrane in rheumatoid arthritis)

Gituin, D., Graig, J. M., and Janeway, C. A. (1957) Amer. J. Path., 33, 55 (Studies on the nature of fibrinoid in the collagen diseases)

GoRMSEN, J. (1970) Scand. J. Haemat. 7, 261 (Fibrinolytic activity and haemagglutination inhibition immunoassays)

- BACH ANDerSEn, R., AND FedDersen, C. (1971) Arthr. and Rheum., 14, 503 (Fibrinogen-fibrin breakdown products in pathologic synovial fluids.

RidDle, J. M., BLuHM, G. B., AND BARNHARDT, M. J. (1965) J. reticuloendoth. Soc., 2, 420 (Interrelationships between fibrin, neutrophils and rheumatoid synovitis)

RYGÅRD, J., AND OLSEN, W. (1969) Acta path. microbiol. scand., 76, 146 (Interference filters for improved immunofluorescence microscopy)

TodD, A. S. (1964) Brit. med. Bull., 20, 210 (Localization of fibrinolytic activity in tissues) 